\title{
1 A unified theory of distraction in human perceptual, cognitive and
}

\section{2 economic decision-making}

3

4 Vickie $\mathrm{Li}^{1 *}$, Elizabeth Michael ${ }^{2}$, Jan Balaguer ${ }^{1}$, Santiago Herce Castañón ${ }^{1}$ and Christopher

$5 \quad$ Summerfield ${ }^{1}$

6

$7 \quad{ }^{1}$ Department of Experimental Psychology

8 University of Oxford

915 Parks Road

10 Oxford, UK

11

12

${ }^{2}$ Department of Psychology

13 University of Cambridge

14 Downing Street

15 Cambridge

16 CB2 3EB

17

18 *Corresponding author: chui.li@psy.ox.ac.uk 


\section{Abstract}

20 When making decisions, humans are often distracted by irrelevant information. Distraction has

21 different impact on perceptual, cognitive and value-guided choices, giving rise to well-described behavioural phenomena such as the tilt illusion, conflict adaptation, or economic decoy effects. However, a single, unified model that can account for all these phenomena has yet to emerge.

24 Here, we offer one such account, based on adaptive gain control, and additionally show that it 25 successfully predicts a range of counterintuitive new behavioural phenomena on variants of a 26 classic cognitive paradigm, the Eriksen flanker task. We also report that BOLD signals in a dorsal 27 network prominently including the anterior cingulate cortex (dACC), index a gain-modulated 28 decision variable predicted by the model. This work unifies the study of distraction across 29 perceptual, cognitive and economic domains.

30

31 Keywords: decision-making; cognitive control; tilt illusion; gain control; anterior cingulate cortex. 
Introduction

Decisions about sensory signals, cognitive propositions, or economic prospects are often made in the context of competing or distracting information. Consider the following everyday situations: you are judging whether a painting hangs straight on the wall, but the nearby pictures are hung askew; you are waiting at a red stop signal, but the car in front decides to jump the light; you are contemplating the purchase of a new watch, but it is displayed next to a range of more elegant but unaffordable models. In each of these situations, the best decisions will be made by ignoring the distracting sensory signals (the competing picture frames, vehicles, or watches) and focussing exclusively on the choice-relevant information. This normative contention can be formalised in a variety of ways, for example via the notion that rational choices should be independent of irrelevant alternatives ${ }^{1,2}$ or that sensory signals should be weighted lawfully by their reliability and relevance to the choice at hand ${ }^{3-6}$.

Nevertheless, empirical observations suggest that humans decision-makers are unduly influenced by distracting information. Consider a generic problem in which a target stimulus $X^{i}$ and distracters $X^{j}$ occur at fixed locations $i$ and $j$. In this general formulation, decision values $X$ may be perceptual features (such as the tilt of a grating) or economic attributes (such as the quality of a consumer product) that are to be evaluated or categorised. Humans show systematic biases that reflect the influence of the distracters on decisions about the target. For example, vision scientists have long studied the "tilt illusion", in which the reported orientation of $X^{i}$ (e.g. a central grating) is repulsed away from the mean tilt of $X^{j}$ (surrounding gratings with similar but nonidentical tilt; Fig. 1a $)^{7}$. In cognitive psychology, the influence of distracter items is usually studied with a view to understanding the attentional or control mechanisms that allow information to be selected in the 
57 face of conflict. For example, in the classic Eriksen flanker task, observers classify a target stimulus

58 (e.g. a central arrow) that is flanked by distracters (e.g. arrows pointing in compatible or

59 incompatible directions) $)^{8,9}$. It is ubiquitously observed that incompatible flankers incur a cost, and

60 compatible flankers a benefit, in response times (RTs) and accuracy relative to a neutral condition

61 (Fig. 1b). Finally, behavioural and neural economists have charted the irrational influence that a

62 decoy alternative of value $X^{j}$ has on choices between two choice-relevant prospects $X^{i+}$ and $X^{i-}$,

63 where $X^{i+}>X^{i-10-13}$. A common finding is that rational choices (i.e. for $X^{i+}>X^{i-}$ ) initially decline

64 as $X^{j}$ increases in value, but then increase sharply as $X^{j}$ comes to approximately match the other

65 two items in value (Fig. 1c). 

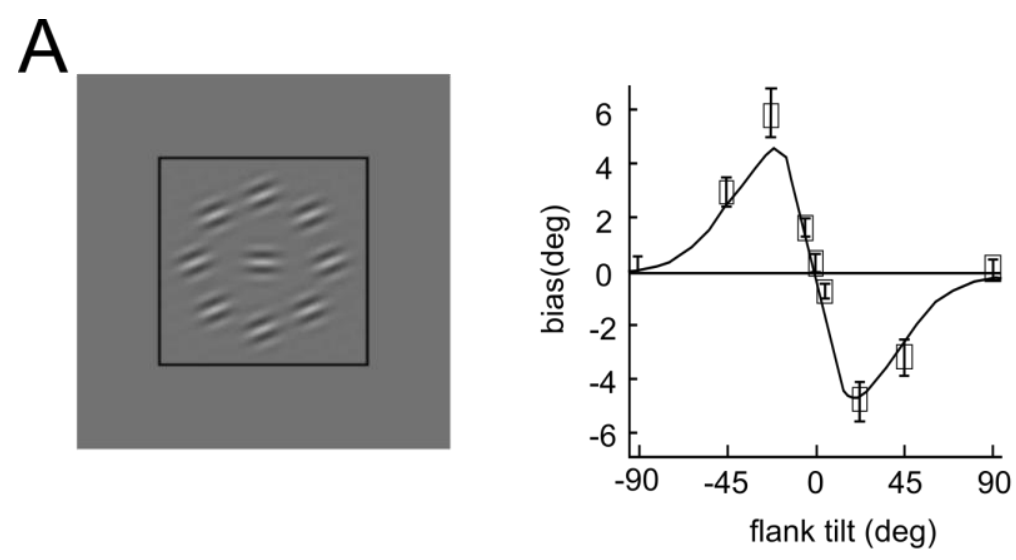

\section{B}
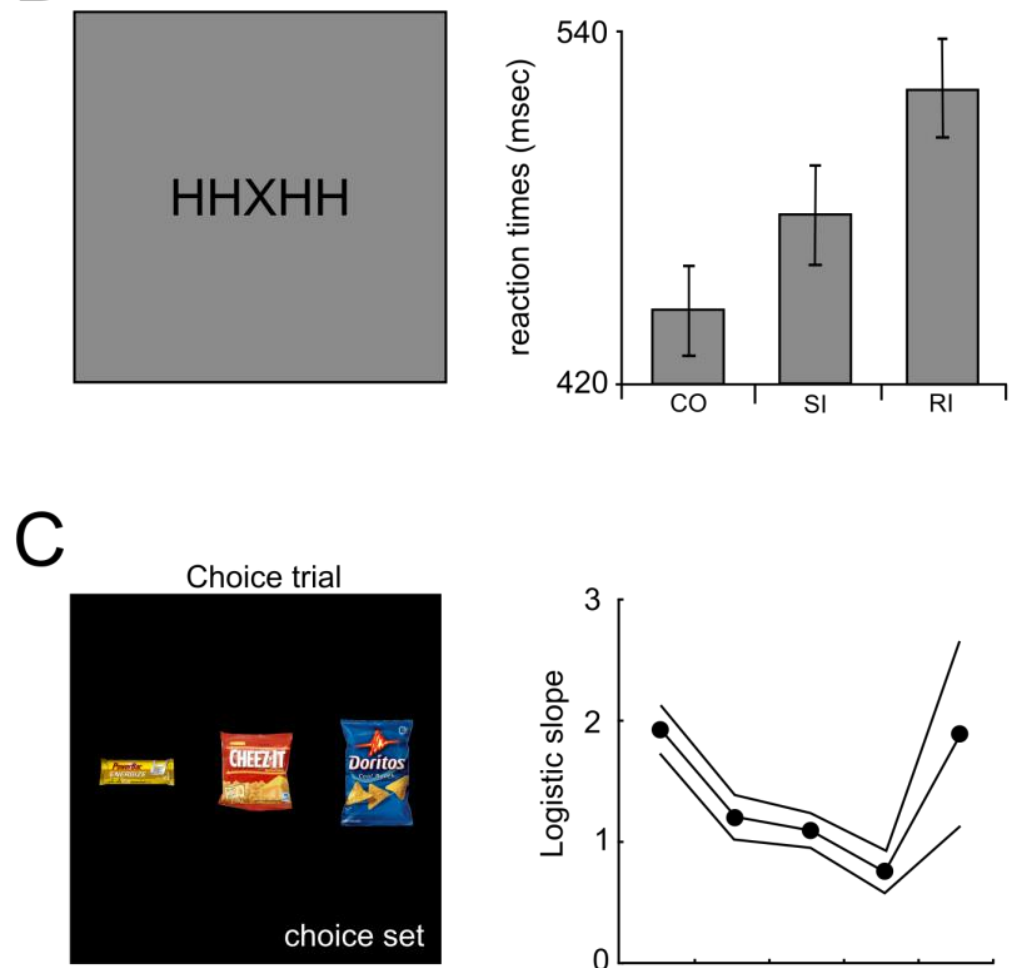

66
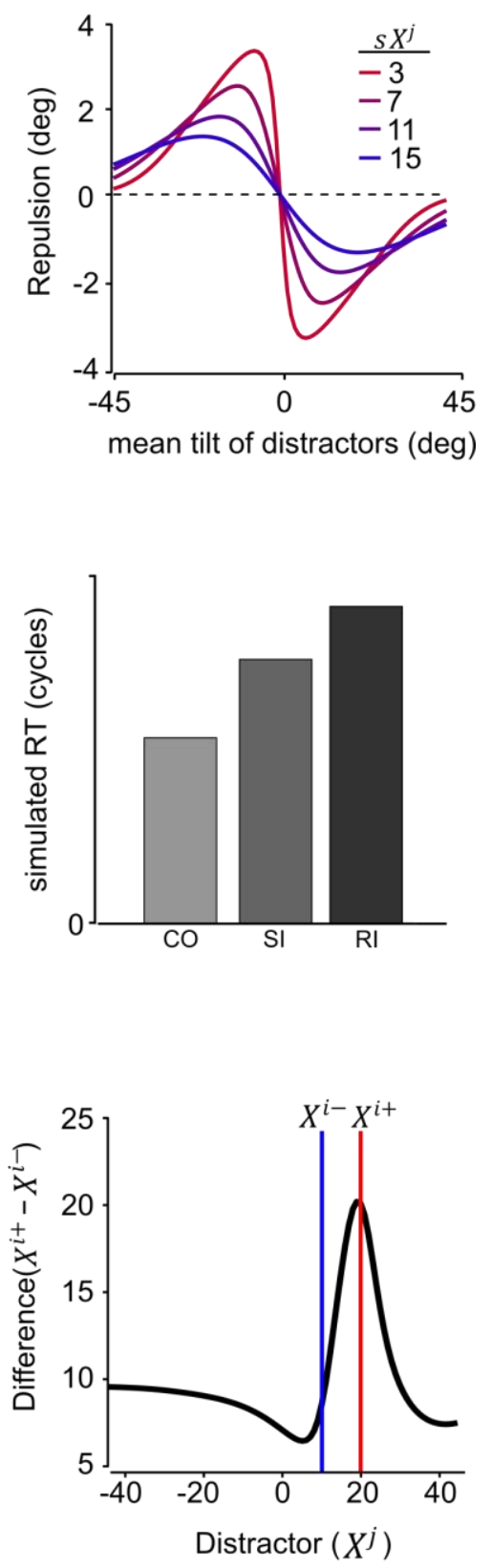

Figure 1. The effect distraction across perceptual, cognitive and economic domains. (A) Left panel: subjects were asked to discriminate the tilt (relative to horizontal) of a central Gabor surrounded by tilted distracters. Middle panel: participants are biased to report the target as more clockwise when the flankers were counterclockwise and vice versa (the "tilt illusion", middle panel; images adapted from Solomon \& Morgan, 2006, permission pending). Right panel: simulation of the adaptive gain model recreates the tilt illusion and further predicts that the magnitude of the bias is modulated by flanker variance; coloured lines reflect flanker standard deviation from low (red) to high (blue). (B) Left panel: In the Eriksen flanker task, participants respond with a key press to a central letter while ignoring the flankers. Middle panel: response times are the fastest on CO ('congruent') trials, then the SI ('Stimulus Incongruent') trials, and slowest on RI ('Response incongruent') trials; Middle panel; adapted from van Veen \& Carter, 2002, permission pending). See methods for details of how CO, RI and SI trials were defined. Right panel: the adaptive gain model predicts the same pattern of reaction time across the three conditions. (C) Left panel: participants chose the most preferred of three food items. Middle panel: increasing the value of the least-preferred item reduces the choice efficiency (i.e. probability of choosing the highest-valued target) as the normalised distractor value increases, shown by logistic slope from fitting logistic choice functions on humans choice. Using the adaptive gain model, we simulated the subjective difference between two tilts (blue and red line) as a function of a third distracting tilt (x-axis). The subjective difference is first reduced and the increased in a qualitatively similar fashion. 
In the fields of psychology, economics, and neuroscience, diverse theoretical proposals have been offered to explain the cost that distracters incur during decision-making. These include models that describe how control systems detect and resolve conflict among inputs ${ }^{14,15}$, accounts that emphasise inhibitory interactions among competing sensory neurons, or favour a normalisation of stimulus values by a local average or range $\mathrm{e}^{10,16-18}$, and Bayesian accounts that model spatial uncertainty among targets and distracters ${ }^{19-21}$, or that assume strong priors on the compatibility of decision information ${ }^{21}$. These accounts disagree about the computational mechanisms involved, the neural processing stages at which the cost of distraction arises, and about the brain structures that are recruited to protect decisions against irrelevant information. For example, divisive normalisation mechanisms may occur in sensory neurons in visual cortex ${ }^{16}$, or amongst value representations in the orbitofrontal cortex ${ }^{22}$, whereas the control systems that detect and resolve conflict have been attributed to medial and lateral prefrontal structures ${ }^{14}$. As such, the field currently lacks a single, unified theory that can account for the effect of distraction on human decisions, or an integrated neural account of its implementation across perceptual, cognitive and economic domains.

84 The goal of the current paper is to offer such an account. We begin with a simple computational model that is motivated by past work showing that contextual signals determine the gain of processing of consistent (or "expected") features during decision-making tasks ${ }^{23}$. Our model, which is described here at the level of neural population codes, proposes that contextual signals sharpen the tuning curves of neurons with compatible preference for decision-relevant features, and is motivated by a large literature emphasising the need for adaptive gain control in the service

90 of efficient coding 24,25 . Using computational simulations, we first show that the model can 91 recreate qualitatively two classic phenomena in very different domains - perceptual choice (the 
tilt illusion) and economic choice (decoy effects). Next, we turn our attention to a task that has been a mainstay of cognitive studies of distraction - the Eriksen Flanker task. We built novel variants of the task in which the statistics of the flankers, and the difference between target and the decision bound can vary across conditions. Our simulations show that the model predicts a range of striking, counterintuitive behavioural findings, including "reverse" compatibility effects (where fully-visible, compatible flankers actually hinder, rather than help, behavioural performance). Over 4 behavioural experiments involving human participants, we validate these predictions, using visual stimuli defined by both tilt and colour. Finally, we use functional brain imaging to show that the modulatory influence on decision signals predicted by the model correlates with BOLD signals the dorsal anterior cingulate cortex (dACC) and interconnected structures, where neural signals have variously been implicated in the context-sensitive encoding of action values ${ }^{26}$, and the expected value of cognitive control ${ }^{27}$. We show how our framework, which is not wholly inconsistent with either account, can bring together diverse views concerning the function of this controversial brain region ${ }^{28}$.

\section{Results}

Our adaptive gain model is based on a framework that was previously developed to understand

110 how humans performing perceptual decision-tasks adapt to the context provided by information

111 that is proximal in space and time ${ }^{23,29}$. Inputs arrive at a population of $n$ neurons each

112 characterised by a Gaussian tuning curve centred on its preferred feature value $\theta_{k}$. Each neuron $k$

113 responds to the target stimulus $X^{i}$ with rate $R_{k}=f\left(X^{i} \mid \theta_{k}, \sigma_{k}\right)$, where $f(X \mid \theta, \sigma)$ denotes the 114 probability density function of the normal distribution with mean $\theta$ and variance $\sigma^{2}$. 
116 The estimated output of the neural population is then decoded into a subjective percept of the

117 target $\widehat{X^{\imath}}$ by weighting the population activity $R$ by the corresponding feature values $\theta$.

$$
\widehat{X^{\imath}}=\sum_{k=1}^{n} R_{k} \cdot \theta_{k}
$$

120 When the gain is uniformly spread across the feature space (i.e. the tuning widths $\sigma_{k}$ for all 121 neurons are equal) this approach faithfully decodes each input to its original feature value. 122 However, our model proposes that the context provided by the distracters modulates the 123 sharpening of neuronal tuning, with a tuning width envelope that matches the inverse distribution 124 of contextual features $X^{j}$ with mean $m X^{j}$ and standard deviation $s X^{j}$.

$$
\sigma_{k}=\sigma^{\max }-f\left(\theta_{k} \mid m X^{j}, s X^{j}+\varepsilon\right) \cdot n
$$

128 In other words, neurons with preferred orientation that matches $m X^{j}$ have the sharpest tuning 129 curves, and these tuning curves are even sharper if the flanker variance $\left(s X^{j}\right)$ is low (see Fig. 2a).

130 In equation (2), $\sigma^{\max }$ denotes the maximum tuning width in the population, and $\varepsilon$ is a noise 131 parameter. In all model fitting procedures below, we allow $\varepsilon$ to vary as a free parameter, 132 recovering average values of $\sim 5^{\circ}$, which concurs with previous accounts ${ }^{30}$. 
A
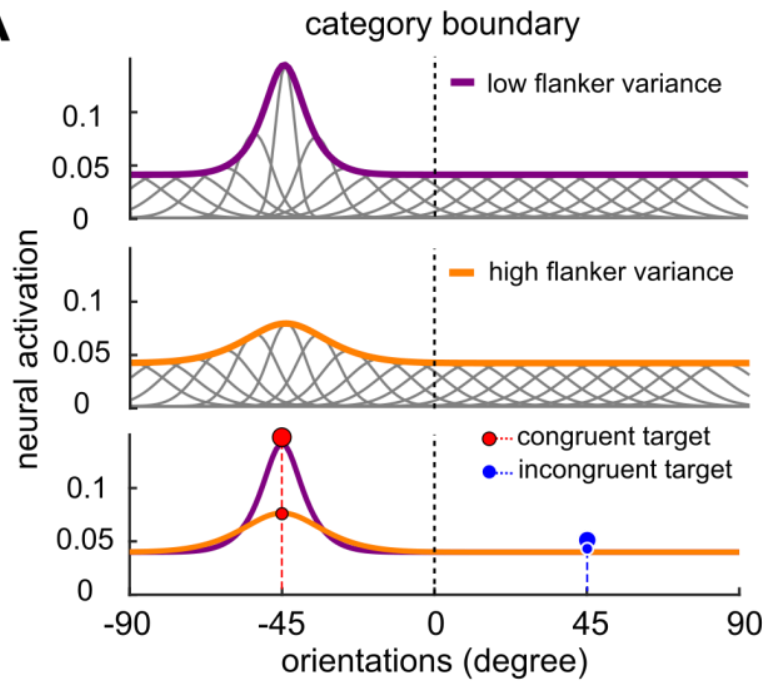

C

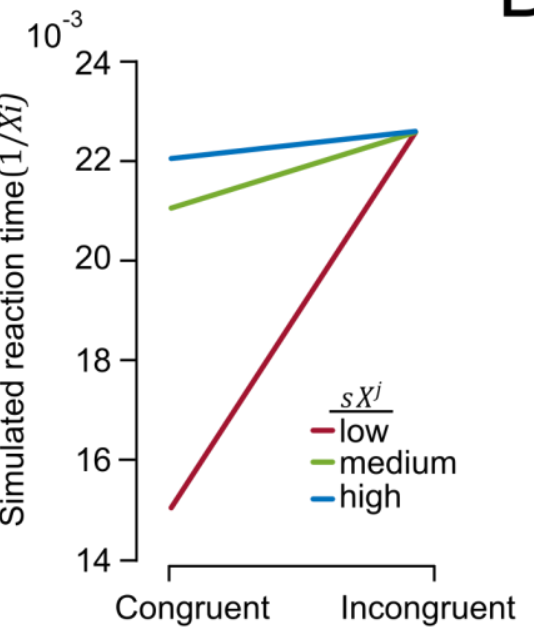

D

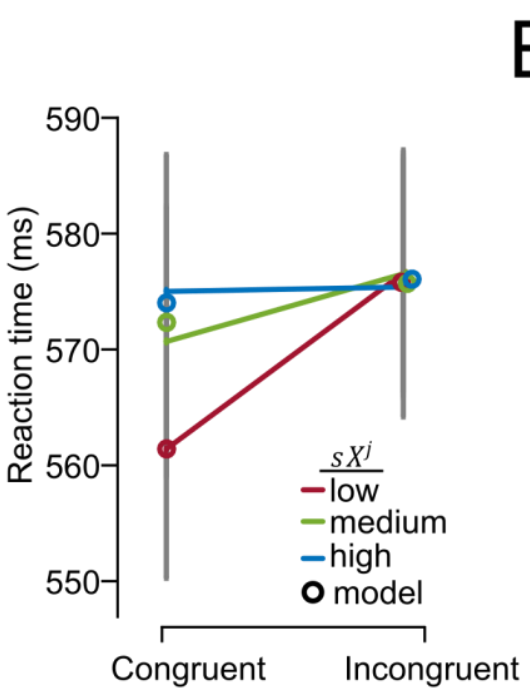

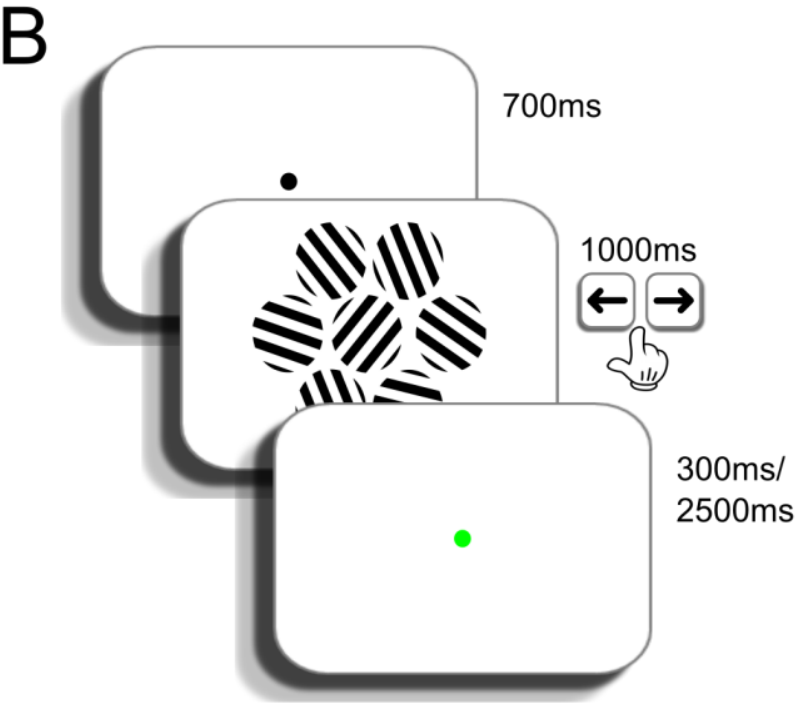

$E$

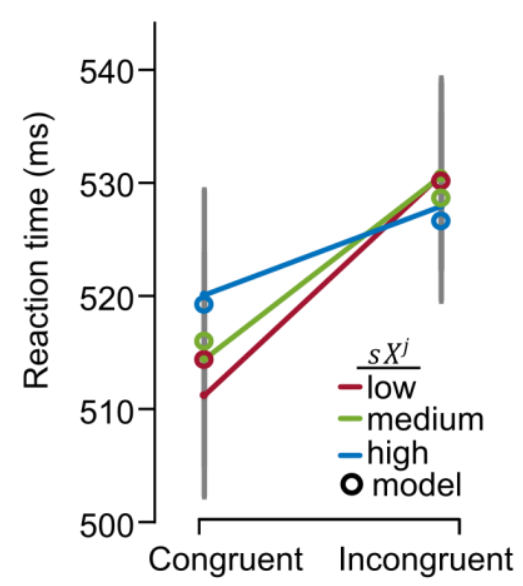

Figure 2. Adaptive gain model of the Eriksen flanker task. (A) Illustration of the adaptive gain model as applied to Exp.1. The model assumes that the population width tuning envelope is governed by the trial flanker mean ( $m X^{j}$ ) and trial flanker variability $\left(s X^{j}\right)$. Congruent targets (red circles) receive higher neural gain than incongruent targets (blue circles), leading to faster RTs. The model further predicts an interaction between congruency and flanker variability. Congruent targets receive high neural gain under low flanker variability trials (large circles) than high flanker variability trials (small circles) while incongruent targets under the two flanker variability trials received the same, low level of neural gain, meaning that flanker variability has no influence on RT for incongruent targets. (B) Experiment 1. Participant first saw a fixation dot, followed by an array that contained a central target and 6 surrounding flankers. They responded whether the central target was clockwise or counterclockwise to the vertical axis, receiving feedback after each response. (C) Simulation of RT (1/| $\left.\widehat{X^{\imath}} \mid\right)$ using the adaptive gain model for 3 levels of flanker variance ( $s X^{j}$ - in coloured lines) and congruency (x-axis). (D - E) Human reaction time pattern under three levels of flanker variance and congruency condition in Exp. 1a (Panel D) and Exp.1b (Panel E). Coloured circles represent the fitted mean reaction time from the adaptive gain model. 
We first show how the model explains the both tilt illusion in perceptual choice tasks and decoy effects in economic choice tasks. In Fig. 1a, we plot the tilt bias over visual angle $\theta \in\{-90,-89 \ldots 90\}$ predicted by the model as a difference of subjective and objective estimates of the target $\widehat{X^{\imath}}-X^{i}$ for different values of $m X^{j} \in\{-45,-44 \ldots 45\}$ and $s X^{j} \in\{3,7,11,15\}$. The model predicts that subjective estimates are repulsed away from the mean flanker value, as described in numerous previous studies ${ }^{7,31}$, and additionally that the strongest repulsion effect occurs when the flankers are consistent, i.e. are drawn from a distribution with low dispersion. This repulsion of the subjectively decoded values from their objective counterparts occurs when the target feature close to, but is not identical to, the mean of the distracters (i.e. the location of sharpest tuning), because the variable tuning profile induces a skew in the population activity over features $\theta_{k}$ (see Fig. S1 for a more detailed explanation). To model economic decoy effects, we envisage a choice between two prospects of value $X^{i+}$ and $X^{i-}$ (where $X^{i+}=20$ and $X^{i-}=10$ in arbitrary units) that is made in the context of distracters with average value $X^{j}$. We plot the difference in their corresponding subjective estimates $\hat{X}^{i+}-\hat{X}^{i-}$ as a function of $m X^{j}$, observing a pattern with a striking qualitative resemblance to that reported previously ${ }^{10}$ (Fig. 1b). Again, the model's ability to predict this counterintuitive pattern comes from the repulsive effect induced by differential tuning across feature space. The model predicts that as the value of the decoy $m X^{j}$ increases, repulsion is first strongest towards $X^{i-}$ (leading to a reduced preference for the best option $X^{i+}$ ) but then, as the decoy approaches the two items in the choice set, repulsion is maximal for $X^{i+}$, reversing this effect. In further simulations, we systematically varied both the distance between $X^{i+}$ and $X^{i-}$, and $s X^{j}$, we were able to capture the two-dimensional pattern of multi-alternative choice data described in a different study involving abstract shapes associated with different economic values ${ }^{11}$ (Fig. S2). 
160 Next, we used our model to simulate performance on a new variant of the flanker task that

161 involves categorising a central grating $X^{i}$ tilted at $-45^{\circ}$ from vertical, in the face of flanking gratings

162 that are on average tilted in a compatible $\left(m X^{j}=-45^{\circ}\right)$ or incompatible $\left(m X^{j}=+45^{\circ}\right)$ fashion.

163 Fig. 2c illustrates predictions from the adaptive gain model under two orthogonally varying

164 factors: compatibility and flanker variability. Because flanker effects for fully-visible stimuli are

165 strongest for response times, we plot the inverse model output $1 /\left|\widehat{X^{\imath}}\right|$ as a proxy for RT (this is

166 equivalent to assuming a ballistic evidence accumulation process; see equation (1) and methods).

167 As can be seen, the model predicts a compatibility effect: faster RTs for trials where the target and

168 distracters were of congruent sign. However, it also makes a new, testable prediction: that as $s X^{j}$

169 (flanker variance) decreases, response times should be reduced on compatible trials but remain

170 the same on incompatible trials (Fig. 2c). This occurs because on compatible trials (flankers at -

$17145^{\circ}$ ), more gain is allocated to the target feature when the variance of the distribution of flanker

172 orientations is lower. However, the gain allocated to incongruent targets (flankers at $+45^{\circ}$ ) is

173 negligibly different across different flanker variance levels since the neural gain they received are

174 similar at the tail of the gain distribution, and so the model predicts that flanker variance should

175 not affect performance on incongruent trials (Fig. 2a). By contrast, classic models propose that

response conflict varies with the amount of crosstalk interference among responses ${ }^{14}$. These

177 models predict that heightened flanker variance should have equal impact on compatible and

178 incompatible trials (see Methods for model details).

180 We tested this prediction in Exp. 1 by asking healthy human participants to judge, relative to 181 vertical, the tilt of a single target grating surrounded by 6 flanking distracter gratings (Fig. 2b). The 182 orientation of the target grating $X^{i}$ and the mean of the flankers $m X_{j}$ were set to $\pm 45^{\circ}$ and the 183 standard deviation of the flankers $s X^{j}$ was varied at three levels: $\left\{0^{\circ}, 15^{\circ}, 30^{\circ}\right\}$ in Exp. 1a $(n=37)$ 
and $\left\{5^{\circ}, 10^{\circ}, 15^{\circ}\right\}$ in Exp. 1b $(\mathrm{n}=36)$. In both experiments, we observed that flanker variance modulated RTs on compatible trials (Exp. 1a: $F_{1.91,68.6}=9.041, p<0.001$; Exp. 1 b: $F_{1.91,68.86}=20.16$, $p<0.001$ ) but not on incompatible trials (both $p$-values $\geq 0.09$ ). This finding was qualified by an interaction between compatibility and flanker variance (Exp. 1a: $F_{1.83,65.79}=12.14, p<0.001$; Fig. 2d Exp. 1b: $F_{1.95,70.06}=20.32, p<0.001$; Fig. 2e). Because previous work has shown that the ratio of compatible to incompatible flankers can modulate performance ${ }^{32}$, we repeated this analysis 190 limited to those trials where all flankers fell on the compatible/incompatible side of the boundary, 191 finding a similar interaction for Exp. 1a $\left(F_{1.83,66.04}=12.59, p<0.001\right)$ and Exp. 1b $\left(F_{1.65,57.66}=15.77\right.$, $p<0.001)$

We fit our adaptive gain model to the data, and compared its predictions to those of a model proposing that RT depends on response conflict alone. The fits for the gain model (coloured circles) are shown superimposed upon the human data in Fig. $\mathbf{2 c}$ and $\mathbf{2 d}$. We compared the models head-to-head by computing mean-squared error (MSE) in RT across conditions on half of the data (even trials), after estimating parameters from an independent dataset (odd trials). Bayesian model selection showed that the adaptive gain model fit the human data more closely than the conflict model, with exceedance probabilities for the adaptive gain model of 0.95 in Exp.

1a and 0.72 in Exp. 1b. We also compared a version of the gain model in which the contextual modulation was driven by both target and distracters; this model yielded both qualitatively and quantitatively similar results to the original gain model ( $p>0.2$ for both Exp. 1a and $\mathbf{1 b}$ ).

205 Next, we moved beyond the simple case in which $X^{i}$ and $m X^{j}$ fell equidistant the category boundary to a more complex design where they could vary independently around vertical at $207\left\{ \pm 15^{\circ}, \pm 30^{\circ}, \pm 45^{\circ}\right)$, and $s X^{j}$ could once again vary at 3 levels $\left\{0^{\circ}, 15^{\circ}, 30^{\circ}\right\}$. In this case, the model 
208 makes several predictions, some of them highly counterintuitive. Firstly, it predicts that there 209 should be no main effect of congruence on RTs. In other words, the predicted inverse decision 210 values $1 /\left|\hat{X}_{i}\right|$ (or equivalently, unscaled RTs) are identical whether the target $\left(X^{i}\right)$ and flanker 211 mean $m X^{j}$ are of the same and different sign. Secondly, the model predicts the existence of 212 strong "reverse compatibility" effects under specific circumstances: there will be a 213 disproportionate cost on congruent trials when the target $X^{i}$ is closer to the category boundary 214 than the mean of the flankers $m X^{j}$, e.g. when $X^{i}=15$ and $m X^{j}=30$ or $m X^{j}=45$ (Fig. 3a 215 upper left corner of each plot), and that this effect should diminish with increasing flanker 216 variance (panels). Finally, although the model indicates that RTs will be dominated by the distance 217 between $X^{i}$ and the category boundary, it predicts that in the specific case where $X^{i}$ and $m X^{j}$ are 218 both close to vertical, this cost will be strongly attenuated. 
A

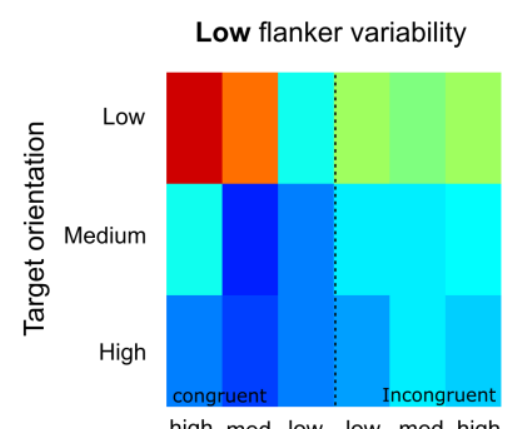

B

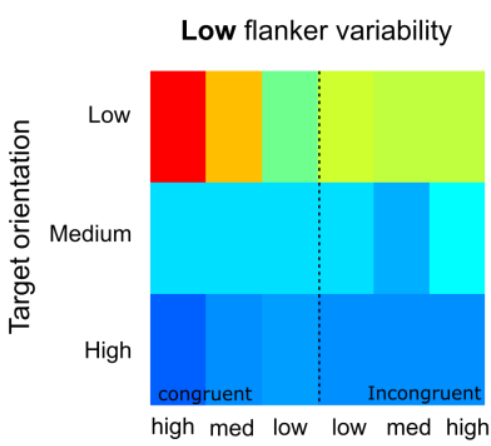

C

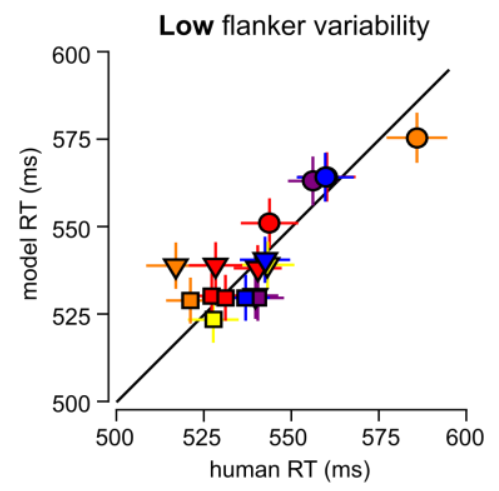

Human

Medium flanker variability

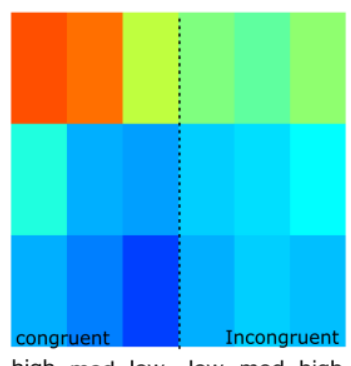

high med low low med high

Flanker mean orientation

\section{Model \\ Medium flanker variability}

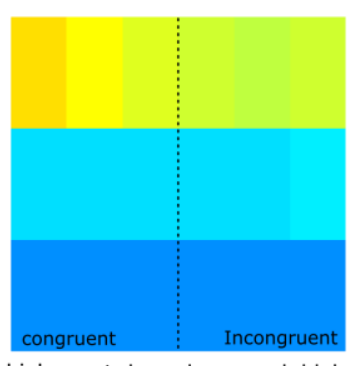

high med low low med high

Flanker mean orientation

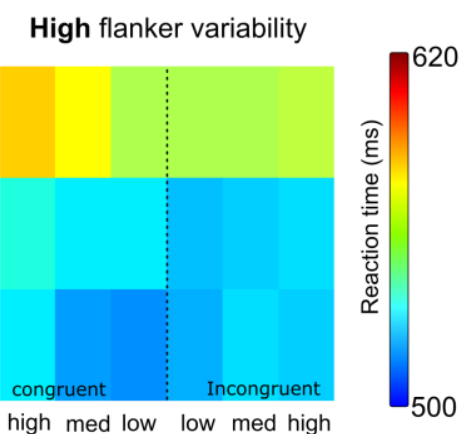

high med low low med high

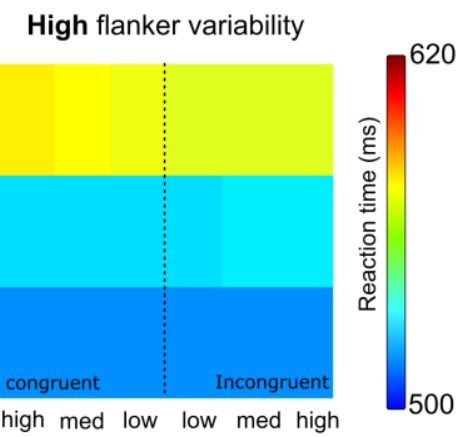

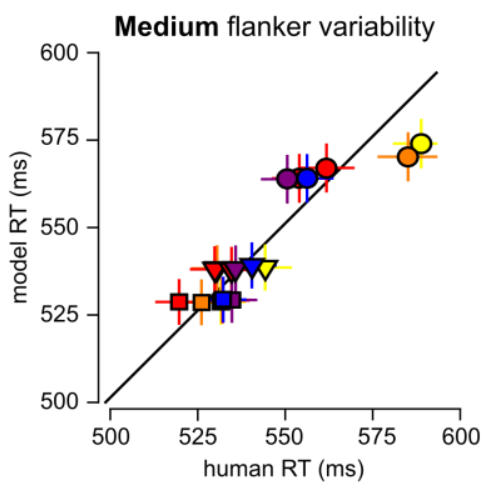
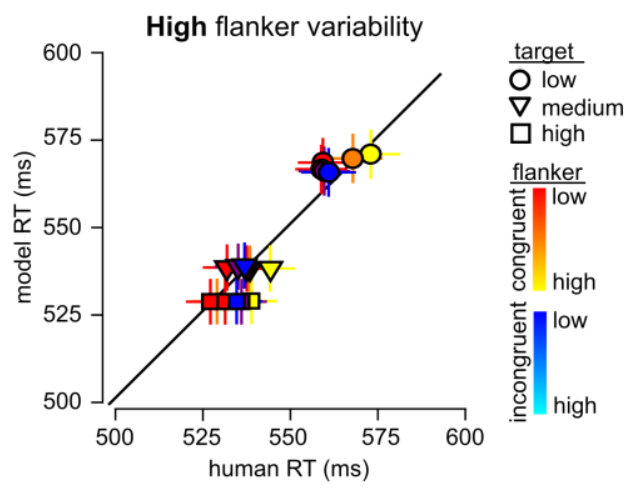

Figure 3. 4 way interactions (A) Surface plots showing the mean RT pattern in humans under different conditions (3 levels of target orientation $\times 3$ levels of flanker mean orientation x congruency $\times 3$ levels of flanker variability). Warmer colours correspond to longer reaction time. There is an overall cost when the target is close to the category boundary (top row of each surface plot). There is an additional cost when these targets were flanked by congruent flankers that are further from the boundary (top left corners of each subplot). This cost is radically reduced when the flankers are both congruent and close to the boundary (c.f. third most column, top row). The introduction of higher flanker variability reduces these additional costs in those conditions (overall faster RT across surface plots). (B) Fitted mean reaction time pattern from the adaptive gain model. (C) Mean ( \pm SEM) RT in humans were cross-plotted against the fitted mean ( \pm SEM) model RT for each condition. Warm colours (Red to yellow) correspond to levels of mean orientation from congruent flankers. Cold colours (Blue to cyan) correspond to levels mean orientation from incongruent flankers $\left|m X^{j}\right|$. Different shapes correspond to 3 levels of target decision variable $\left|X^{i}\right|$. Similar to upper plots, slower RT occurs specifically on trials where the target is close to the boundary and flankers are both congruent and have a mean orientation that is further from the category boundary. 
220 We tested these predictions using the flanker paradigm on two new cohorts of participants, one of

221 which (Exp. 2a, $n=28$ ) performed the tilt categorisation task described above, except with the full

2226 (target mean) $\times 6$ (flanker mean) $\times 3$ (flanker variance) design. Another (Exp. 2b, $\mathrm{n}=30$ )

223 performed a task with the same design that involved judging the colour of a central circle (red vs.

224 blue) surrounded by distracting flankers that varied continuously in colour from red to blue.

225 Results from the two experiments were qualitatively very similar (see Fig. S3, S4 for separate data)

226 and so after normalising the feature values (tilt, colour) to an equivalent scale in the range $[-1,1]$

227 we collapsed over them for display purposes. All three model predictions were strongly present in

228 the human RT data (Fig. 3a-c). Firstly, splitting trials naively into compatible and incompatible,

229 there was no overall significant difference in RT, either in the combined cohort $\left(t_{57}=1.14, p=\right.$

230 0.26) or separately for each experiment (Exp. 2a: $p=0.79$; Exp. 2b: $p=0.17$ ). Secondly, we see a

231 strong cost on congruent trials when the flanker mean is further from the boundary than the

232 target mean, as demonstrated by an $X^{i} \times m X^{j}$ interaction $\left(F_{3.72,208.23}=17.48, p<0.001\right)$ that was

233 much weaker on incongruent trials $\left(F_{3.39,212.25}=2.47, p=0.049\right)$. This difference was qualified by a

234 reliable three-way $\left|X^{i}\right| \times\left|m X^{j}\right| \times$ congruence interaction on human $\mathrm{RTs}\left(F_{3.54,198.12}=14.62, p\right.$ $235<0.001)$

237 The analyses described thus far pertain to RT data. In a final analysis of Exp.2 we examined 238 choices, using a previously-described approach based on probit regression to assess the weight (or 239 influence) that distracters wielded over choices, as a function of whether their tilt was inlying or 240 outlying with respect to category boundary ${ }^{33,34}$. We attempted to predict choices on each trial as 241 follows:

$$
p(C W)=\Phi\left(\beta^{0}+\beta^{1} \cdot X^{i}+\beta_{j}^{2} \cdot X^{j}\right)
$$


245 Where $X^{j}$ is tallied into 8 bins according to its signed feature value (e.g. from most 246 counterclockwise to clockwise, or most red to blue). Plotting the coefficients $\beta_{j}^{2}$ associated with 247 each bin $j$ for model choices (obtained via the fitting procedure above), the model 248 counterintuitively predicts that inlying flankers (those whose feature value falls close to the 249 category boundary) will have a stronger impact on choices than outlying flankers. This occurs 250 because the context-dependent gain field is, on average over all trials, Gaussian with a mean of 251 approximately zero; in other words, the tuning of neurons near the category boundary is on 252 average sharper. When we applied the same analysis to human data, we observed exactly the 253 same pattern, with inlying flankers exerting a more distracting influence on choices (Fig. 4a). 254 Applying the same regression model to predict RTs, the plots of $\beta_{j}^{2}$ for both humans and the 255 model show the characteristic repulsion effect in choices, that is owing to the greater contextual 256 modulation of neural tuning near the category boundary (Fig. 4b). Together, these analyses show 257 that the human data resemble the counterintuitive model predictions over 4 different 258 experimental datasets. 

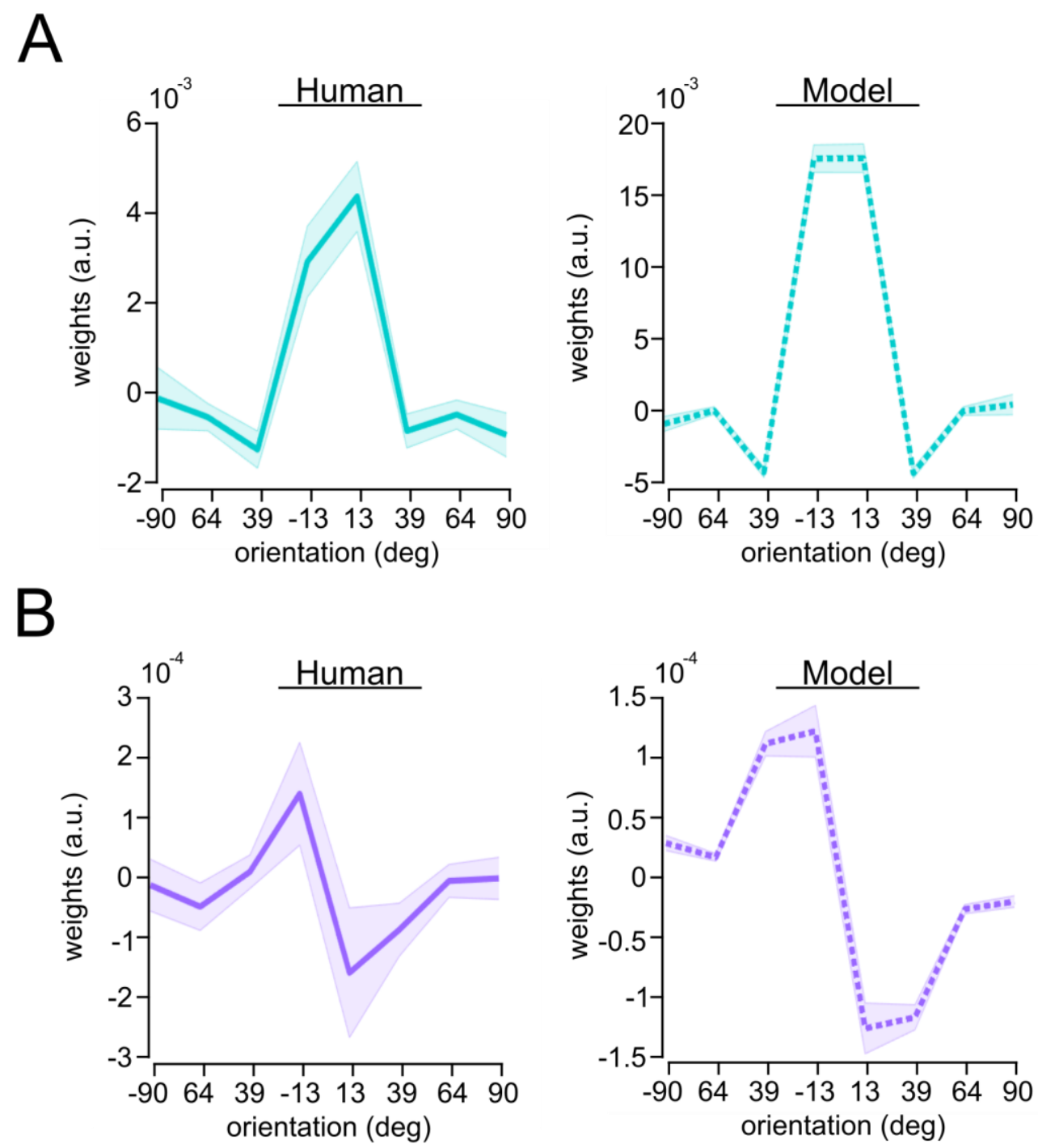

Figure 4. Choice bias driven by the flankers. Flanker tilts sorted into 8 bins from most CCW to most CW and used for predict choice (panel A; using probit regression) and response times (panel B) for both humans (left panels) and the best-fitting model (right panels). The x-axis shows the centre of each tilt bin, and the $y$-axis shows the resulting coefficients. The inverted-U-shape pattern in Panel A reveals that the inlying flankers have a stronger impact on choice than outliers for both humans and model. The response time data reveal the repulsive nature of this effect (Panel B solid purple line; note similarities with Fig. 1a). Higher absolute magnitude of beta weights implies that orientations that are close to the category boundary are more predictive of RT, similar to what was shown on choice in panel A. Shaded areas are the standard error of mean.

Functional brain imaging

263 Established theories propose that a brain network that prominently includes the dorsal anterior cingulate cortex (dACC) is involved in the recruitment of control processes that allow the brain to 
overcome distraction. Across a range of paradigms including the Eriksen Flanker task, the dACC responds with higher-amplitude BOLD signals on incompatible than compatible trials ${ }^{35,36}$, and this effect is accentuated when the previous trial was compatible ('conflict adaptation'), as if the dACC is monitoring for conflict and signalling its onset ${ }^{37}$. However, the dACC is also implicated in decision processes more generally. For example, it signals the level of noise that corrupts an imperative stimulus during perceptual discrimination ${ }^{38}$, and its proximity to a choice point or category boundary ${ }^{39}$; it responds to the relative economic value of an unchosen to a chosen option ${ }^{40-42}$, to the value of switching to a new task or context $\mathrm{t}^{43,44}$, and to the update signals that occur as decision values change ${ }^{45}$. The search for a unifying theory of the dACC has been one of the most challenging and controversial themes in cognitive neuroscience over recent years ${ }^{28,46-48}$.

To assess the role of the dACC and interconnected regions in adaptive gain control, we conducted a new experiment in which $X^{i}, m X^{j}$ and $s X^{j}$ varied parametrically from trial to trial, rather than in a conditionwise fashion. A new cohort of humans (Exp.3; $n=20$ ) performed this task whilst we acquired BOLD signals from across the brain using functional magnetic resonance imaging (fMRI). Behavioural results of this experiment replicated those from Exp.2 (Fig. S5), and so we focussed on neural analysis to test whether brain signals indexed decision information in a way that was predicted by the adaptive gain model. We began by confirming previous reports that the dACC responds more vigorously when a target feature lies closer to a category boundary, i.e. in our experiment, when the target orientation is closer to vertical ${ }^{39}$. We first regressed $\left|X^{i}\right|$ (i.e. proximity of the target to the category boundary) alone against BOLD signals occurring at the time of choice across the entire brain (GLM1). Consistent with previous observations, we observed a negative effect of $\left|X^{i}\right|$ in the dACC (peak: $2,8,54, \mathrm{t}_{19}=9.66, \mathrm{p}_{\mathrm{fdr}}<0.001$ ), as well as the anterior 
$28958, \mathrm{t}_{19}=8.09, \mathrm{p}_{\mathrm{fdr}}<0.001$ see Fig. 5a). Extracting regions of interest from these areas in a leave290 one-out fashion across participants (see Methods), we then plotted how the BOLD signal varied in 291 quartiles of both $X^{i}$ and $X m^{j}$ (GLM2) and compared these signals to the predictions of (i) the 292 adaptive gain model, (ii) an equivalent model with no adaptive gain, i.e. where all simulated cells 293 had equivalent tuning width, and (iii) a model in which BOLD signals were driven by conflict alone 294 (Fig. 5b). We found that the pattern of BOLD signals in all 3 regions closely resembled that 295 predicted by the adaptive gain model, but not the other models (Fig. 5c). Specifically, although 296 BOLD responses were elevated when the $X^{i}$ was close to zero (dACC: $F_{1,19}=52.37, p<0.001$; AIC: $297 F_{1,19}=53.4, p<0.001 ;$ SPL: $\left.F_{1,19}=48.94, p<0.001\right)$, this effect was exaggerated on those trials 298 where $m X^{j}$ was far from zero but of compatible sign (i.e. greater BOLD response in dACC, AIC and 299 SPL on congruent relative to incongruent trials; dACC: $t_{19}=3.03, p=0.0069 ;$ AIC: $t_{19}=2.82, p=$ $\left.300 \quad 0.011 ; \mathrm{SPL}: \mathrm{t}_{19}=2.28, \mathrm{p}=0.034\right)$. No such modulation was observed when $X^{i}$ was far from zero, as 301 predicted by the adaptive gain model. 


\section{A}
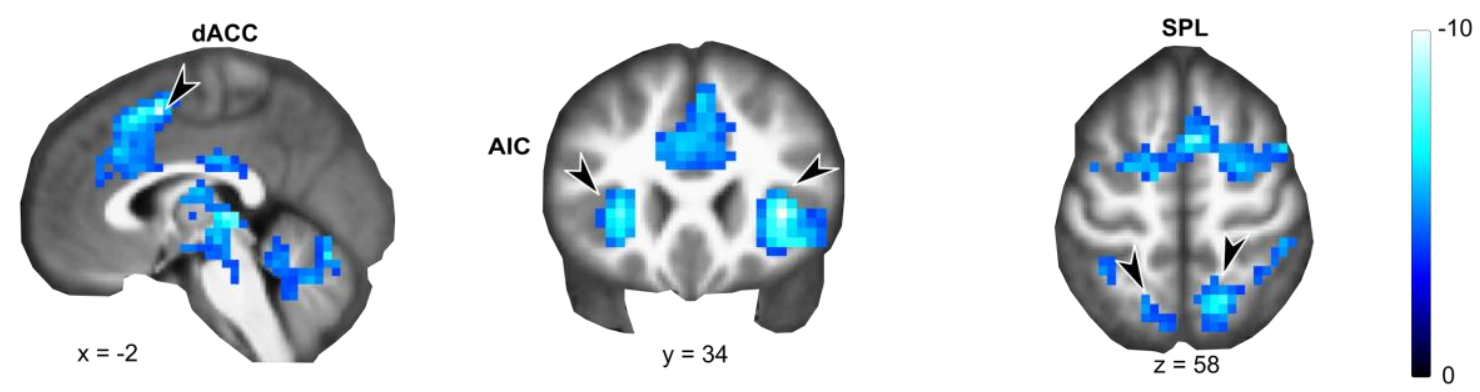

B
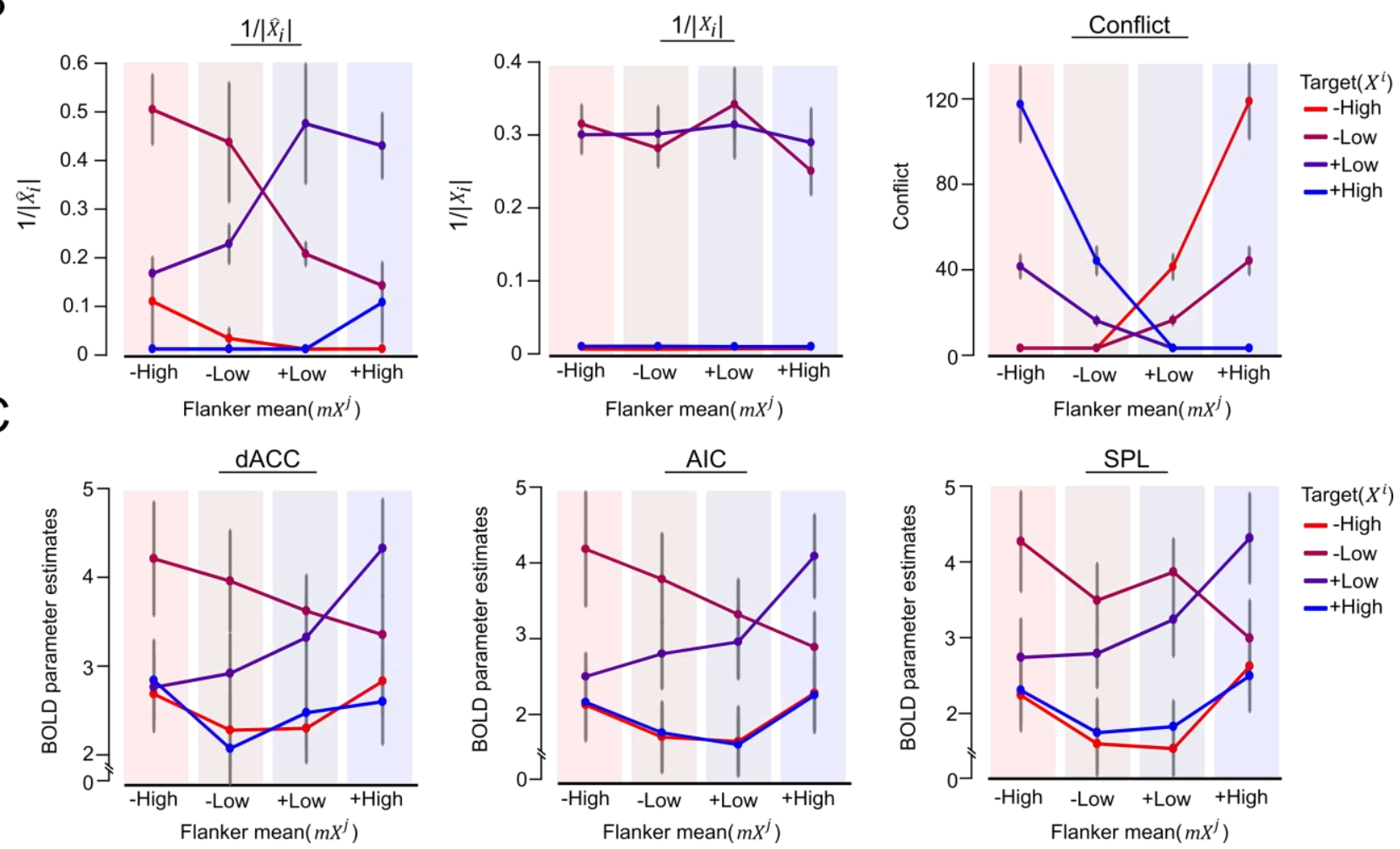

Figure 5. Effect of target orientation on BOLD signal (A) Brain areas correlating negatively with the absolute target decision variable, rendered onto a template brain in sagittal (left panel) and axial (middle and right) slices. Images were generated with an uncorrected threshold of $\mathrm{p}<0.0001$. (B) Parametric modulators $X^{i}$ and $m X^{j}$ were each being split into 4 quartiles (lines for $X^{i}$, x-axis or shaded area for $m X^{j}$ ). Mean predictions $( \pm S E M)$ from different models: Reciprocal gain-modulated decision variable $1 /\left|\hat{X}_{i}\right|$ (left panel). Reciprocal no-gain modulated target decision variable $1 /\left|\hat{X}_{i}\right|$ (middle panel). Estimated conflict was plotted for from each quartile bins (right panel). Coloured lines correspond to 4 levels of $\left|X^{i}\right|$. Shaded coloured background (groupings on $x$-axis) corresponds to 4 levels of $m X^{j}$ (C) Mean BOLD ( \pm SEM) signal beta values for each level of $\left|X^{i}\right|$ and $m X^{j}$ from the quartile bins. Left: dACC, Middle: AIC, and Right: SPL ROls.

This suggests that a gain-modulated decision variable, rather than a conflict signal per se, is driving

the dACC response. However, to quantify and compare the predictions of different models, we 
307 models on the trial RTs. Model estimates $\left|X^{i}\right|$ and conflict computed in different ways (see 308 Methods) from the best fitting parameters are then used to estimate BOLD signals. We computed, 309 within the dACC, AIC and SPL, the posterior probability of the adaptive gain model conditioned on 310 the BOLD signal using Random effects Bayesian model selection from the VBA toolbox ${ }^{50}$, and 311 compared the resulting estimates to those obtained for rival models. Both the exceedance 312 probabilities and the expected frequencies strongly favoured the adaptive gain model over a 313 decision model with no gain modulation, as well as over a family of conflict models (exceedance 314 probabilities for the adaptive gain model in dACC: 0.992; AIC: 0.994; SPL: 0.996; expected 315 frequencies for the adaptive gain model compared to chance level in dACC: $t_{19}=4.11, p<0.001$; 316 AIC: $\mathrm{t}_{19}=4.11, \mathrm{p}<0.001 ; \mathrm{SPL}: \mathrm{t}_{19}=4.76, \mathrm{p}<0.001 ;$ Fig. 6a). In other words, the dACC, along with 317 AIC and SPL, code for a decision signal modulated in precisely the fashion predicted by the 318 adaptive gain model. 
A

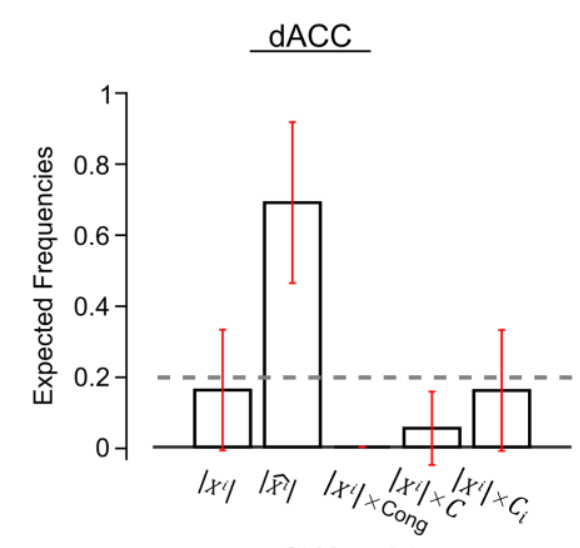

GLM model

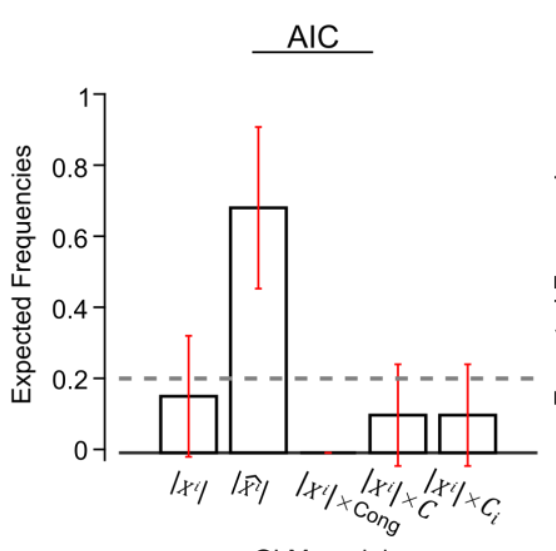

GLM model

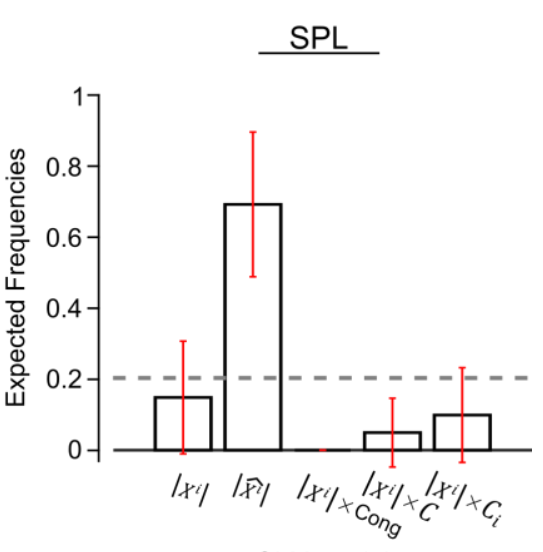

GLM model

B

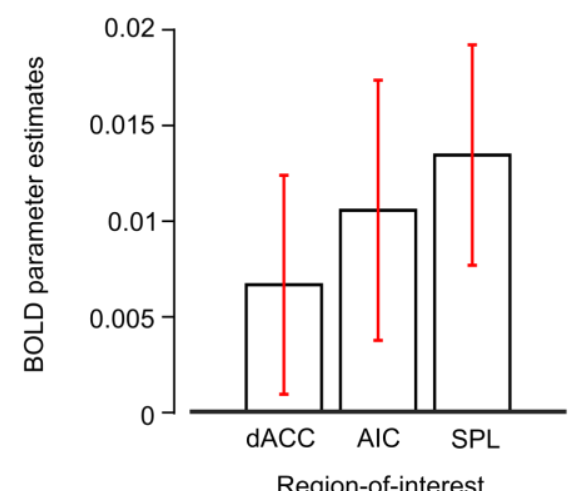

C

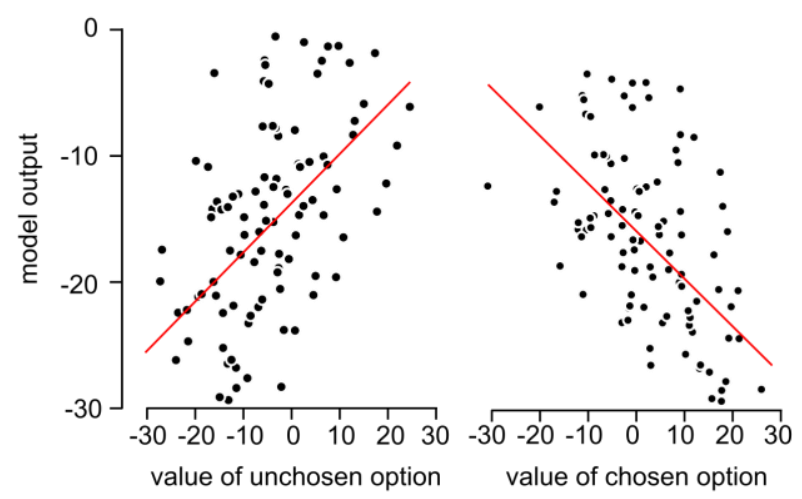

Figure 6. (A) Model comparisons across GLM models. Expected frequencies for each GLM and each ROI were computed using Random effects Bayesian model selection. Grey dotted line displayed the chance level (B) ROI analysis on $\mathrm{dACC}, \mathrm{AIC}$, and SPL on the absolute flanker mean decision variable $\left|m X^{j}\right|$. Bar plots correspond to the mean $( \pm S E M)$ beta estimates for this predictor from GLM5. (C) Simulated (negative) model output as a function of the value of chosen and unchosen option (see methods for details).

321 Finally, we addressed a concern that dACC is simply exhibiting a BOLD signal that correlates with the response production time on each trial ${ }^{51}$. Disentangling these factors is challenging, because (as described above) the model does an excellent job of predicting RTs. Nevertheless, when we included both model output $1 /\left|\hat{X}_{i}\right|$ and RTs as competitive predictors in the model (GLM4), we still recovered a significant activation in the dACC (peak: $2,20,50, t_{19}=5.28, p_{f d r}=0.023$ ), and AIC (left peak: $-30,20,6, \mathrm{t}_{19}=6.09, \mathrm{p}_{\mathrm{fdr}}<0.001$; right peak: $34,24,-6, \mathrm{t}_{19}=5.94, \mathrm{p}_{\mathrm{fdr}}<0.001$ ). In other 
words, the dACC BOLD signal correlates better with the demand predicted by the adaptive gain model than it does with time taken to produce a response on each trial.

How does our model explain previously-reported findings, such as the observation that the dACC responds to conflict ${ }^{52}$, or to the relative value of an unchosen vs. a chosen option during economic choice $^{53}$ ? We have already shown that in the simple version of the Flanker task (c.f. Exp.1a where $\left.s X^{j}=0\right)$, the model predicts a larger output $1 /\left|\hat{X}_{i}\right|$ on incompatible relative to compatible trials.

The model is thus in clear accord with a large literature indicating that dACC BOLD increases when target and distracters are incongruent in a simple version of the Flanker task ${ }^{54}$. We note that as described here, the adaptive gain model computes decision values independently on each successive trial, and thus in its current form would not predict conflict adaptation in the dACC. However, one could reasonably assume that adaptive effects may spill over from one trial to the next, i.e. that neural tuning width will be partly modulated by the previous trial. Under this assumption, the adaptive gain model will successfully predict that responses should be faster on two successive incongruent or two successive congruent trials ${ }^{55}$, just as it successfully account for the observation that during categorisation of a multi-element array, response times are faster if the target array is preceded by a prime array with an equivalent level of feature variance ${ }^{29}$.

However, we also note another facet of our results: that BOLD signals in the AACC, AIC and SPL ROIs correlate negatively with $\left|X^{i}\right|$ but positively with $\left|m X^{j}\right|$ (GLM5; Fig. 6b; dACC: $t_{19}=2.27, p=$ 0.035, AIC: $\mathrm{t}_{19}=3.03, \mathrm{p}=0.003, \mathrm{SPL}: \mathrm{t}_{19}=4.56, \mathrm{p}=0.007$; this effect was also significant at the whole-brain level in voxels within the AIC and SPL, but not dACC). If we consider the target to be a "chosen" option and the flankers as a competing, "unchosen" option, the ensemble of findings 
351 with the decision value associated with an unchosen option (i.e. the flankers) and negatively with

352 the value of a chosen option (i.e. the target). Building on this intuition, we tested more directly the

353 coding of model-predicted value of a chosen and unchosen option in a further simulation in which

354 decision values for two stimuli were drawn randomly and independently from two distributions, 355 and model output was converted to a choice via a softmax function (see Methods). This allowed 356 us to correlate model output (i.e. predicted BOLD) with the value of the chosen and unchosen 357 option, revealing a negative correlation with the former and a positive correlation with the latter, 358 as previously reported ${ }^{56}$ (Fig. 6c). Our model thus unifies a number of disparate accounts that 359 have emphasised a role for the dACC in tasks involving categorising perceptual stimuli, and 360 choosing among economic prospects.

\section{Discussion}

Good choices are based solely on information that is relevant to the choice at hand, and rational agents will successfully ignore distracting signals when making decisions ${ }^{1}$. However, across perceptual, cognitive and economic domains, human participants are observed to deviate from this rational principle. A range of different theories have been proposed to account for human susceptibility to distraction, but thus far, no single model has emerged that can account for phenomena as diverse as visual illusions, susceptibility to conflicting contextual features, or

371 economic decoy effects. Here, we describe one such account. Our adaptive gain model has previously been shown to successfully account for diverse contextual influences in perceptual decision-making, including confirmatory biases in sequential sampling ${ }^{23}$ and priming by second- 
order summary statistics in perceptual categorisation ${ }^{29}$. Here, we show that not only can it account for classical effects of distraction across perceptual, cognitive and economic domains, but also that it successfully predicts a range of previously unreported, counterintuitive findings in a well-studied cognitive paradigm, the Eriksen flanker task ${ }^{8}$.

The effect of distraction is most often modelled under the assumption that irrelevant features are 380 imperfectly filtered during decision-making, driving residual activation that corrupts decisions. 381 When target and distracters prompt conflicting sensorimotor responses, the resulting competition 382 slows response times and increases error rates ${ }^{14}$. A very successful neurocognitive theory proposes that dedicated processing systems have evolved in the primate medial prefrontal cortex that detect this conflict, and that are responsible for mobilising control mechanisms (associated with the lateral prefrontal cortex) to help mitigate the resulting costs ${ }^{57}$. Here, we propose a differing view: one that emphasises the benefits of consistent context rather than the costs of inconsistent context. In the adaptive gain model, contextual features offer guidance as to where to best allocate gain across feature space, ensuring that neurons that code for the most prevalent 389 (or "expected") features have the sharpest tuning and thus provide the most sensitive outputs. 390 The adaptive gain model is thus motivated by the more general view that the nervous system has 391 evolved to code for efficiently for sensory inputs, reducing redundancy by dynamically adjusting 392 the tuning properties of decision-relevant neurons to maximise sensitivity to expected 393 features ${ }^{24,58}$. In the flanker task, thus, the difference between compatible and incompatible trials 394 arises at least in part because of a contextual facilitation mechanism at the decision level, akin to 395 that described in sensory circuits ${ }^{59}$, rather than because of an active cost of response competition. 396 This idea is not without precedent in theories of control. In fact, the notion that a flexible response 
to stimulus conflict is dependent on adaptive expectation mechanisms dates back to the original discovery of conflict adaptation by Gratton and colleagues more than 25 years ago ${ }^{55}$.

We take the opportunity to highlight two major features of our behavioural data that cannot be 401 accounted for by standard accounts that emphasise the cost of conflict alone. Firstly, in Exp.1a-b, 402 we found that a low-variance flanker array hastens response times on congruent trials, rather than 403 prolonging response times on incongruent trials. This is consistent with an account that 404 emphasises the benefit of consistent context rather than the cost of inconsistent context. 405 Secondly, in Exp.2a-b, we observed that the longest response times were in fact observed on compatible trials, not incompatible trials. We replicated this finding across two different classes of visual feature: tilt and colour. According to our model, this cost occurred when the gain field 408 dictated by the context repulsed the target subjectively closer to the category boundary, 409 rendering choices more uncertain. Although such negative flanker effects have been reported 410 with heavily masked stimuli, where they can be explained by differing timecourses of facilitatory 411 versus inhibitory processes ${ }^{60}$, only rarely have such phenomena been reported for fully visible 412 targets and distracters such as ours. Most interestingly, one such report occurred for a modified 413 version of the flanker task where the targets were letters that were parametrically morphed 414 between two possible identities, each corresponding to a possible flanker ${ }^{61}$. This report describes 415 negative flanker effects when the target is most ambiguous, precisely parallelling our findings here 416 for trials with small $X^{i}$ and large $\mathrm{m}^{j}$, and a shift in the psychometric function that occurs with 417 flanker identity in precisely the fashion predicted by our adaptive gain model ${ }^{23}$.

419 Our behavioural findings were echoed in the neural data recorded from dACC, where BOLD signals 
modulated (yet higher) when the distracters mean was congruent but further from the boundary. Without further assumptions, a model based on conflict alone cannot account for these findings. We do not wish to argue that stimulus or response conflict do not ever incur an additional cost to accuracy and response times, or that such a cost is unable to drive the dACC. Nevertheless, in the current study, we found that such an account was not required to explain our data, and that a model embodying this assumption fit our data more poorly.

Our findings present a challenge to extant theories, but we acknowledge that our model is currently incomplete. A large literature implicates the dACC in the mechanisms by which we update the value of actions in dynamically changing environments ${ }^{45,62}$. Our experiments were conducted in stationary settings, and we do not doubt that these regions may play additional roles 432 (potentially also related to gain control) when slower learning about a changing context is required. We also note an important shortcoming in our findings: we were unable to identify 434 differing roles for the dACC, AIC and SPL, which seem to act as one in our study. We think it is 435 likely that our BOLD data are simply indexing the output of a decision process that involves 436 modulation by distracting context, but are unable to make strong claims about the interim 437 processes by which the computations proposed by the model occur. We suspect that exploring 438 the role of adaptive gain control in dynamically-changing environments may shed more light on 439 the differing contributions made by these regions, and we hope to pursue this question in future 440 studies.

\section{Methods}


444 For behavioural studies Exp.1 and 2, human participants were recruited via the online testing 445 platform provided by Amazon Mechanical Turk (Exp. 1a: $\mathrm{n}=37,19$ men, mean age: 31.8 years, SD $446=8.09$; Exp.1b: $\mathrm{n}=36,22$ men, mean age: 31.12 years, $\mathrm{SD}=9.57$; Exp. $2 \mathrm{a}: \mathrm{n}=28,14$ men, mean 447 age: 33.5 years, $S D=13.09$; Exp.2b: $n=30,16$ men, mean age: 33.3 years, SD $=9.5)$. Mind here 448 that the age information was collected by sets of age (e.g. 18-20, 21-30, 31-40 etc.) due to online 449 testing, thus the reported age from Exp. 1a,1b,2a,2b was computed by taking the bin center of 450 each age bin as the approximation of participants' true age. For Exp.3, 20 healthy volunteers (8 451 men, mean age: 24.25 years, SD $=4.61$ ) with normal or corrected-to-normal vision and no history 452 of neurological disorders were recruited to participate. All participants gave informed consent to 453 participate in the study and were compensated at a rate of $\$ 6$ per hour for Exp. 1 and 2 and $€ 10$ 454 per hour for the fMRI scanning session. All experiments were approved and conducted in 455 accordance with the Medical Sciences Inter-divisional Research Ethics Committee (MS IRDREC) 456 ethical guidelines.

\section{Task details}

459 Experiment $\mathbf{1 a}, \mathbf{1} \boldsymbol{b}$ and $\mathbf{2} \boldsymbol{a}$. Each trial began with a fixation dot $700 \mathrm{~ms}$, followed by the 460 simultaneous presentation of target and flankers for $1000 \mathrm{~ms}$. Target and flankers were full461 contrast square-waved gratings with a $180^{\circ}$ phase (5 cycles, and 75 pixels in diameter). The 462 flankers are presented within a circular aperture of the target grating with 1.5 pixels separation 463 with adjacent flankers. The orientation of each individual grating in the flanker surround was 464 chosen form a Gaussian distribution with a particular mean $\left(m X^{j}\right)$ and standard deviation $\left(s X^{j}\right.$; 465 see main text for precise values). To ensure that the sampled orientations matched the expected 466 distribution with the given $m X^{j}$ and $s X^{j}$, resampling of orientation values occurred until the mean 467 and standard deviation of orientations fell within $1^{\circ}$ tolerance of the desired $m X^{j}$ and $s X^{j}$. Within 
468

the stimulus presentation duration, participants had to indicate whether the central grating was clockwise or counterclockwise to the vertical axis by pressing the right and left keyboard arrows respectively. They were instructed to ignore the flanking gratings. Visual feedback was given immediately after their response. Participants completed 6 blocks of 96 trials for Exp. 1a, 10 blocks of 96 trials for Exp. 1b, and 10 blocks of 108 trials for Exp. 2a).

Experiment $\mathbf{2 b}$. While keeping all other aspects of the experiment (e.g. timing) the same, in Exp.2b we substituted the grating stimuli with coloured stimuli from a continuous colour space. Target and flankers were circles (100 pixels diameter per circle; 19 pixels separation with adjacent flanking circles) with hue ranging from blue to red. Participants were asked to indicate whether the central colour circle was more red (right arrow) or blue (Left arrow) while ignoring the surrounding coloured circles. Individual colour values $(c)$ were defined by RGB values using expression: $\mathrm{RGB}=[c, 0,1-c]$ with the category boundary being defined as $c=0.5$ or $\mathrm{RGB}=[0.5,0$, 0.5]. The colour value for the central target stimulus and the flanker mean colour value were drawn from the set $\{0.35,0.4,0.45,0.55,0.6,0.65\}$. To facilitate comparison between Exp.2a and Exp.2b, we recentered the category boundary value to 0 , which means the central target colour values $\left(X^{i}\right)$ can be redescribed as $\pm 0.05,0.1,0.15$ with respect to a category boundary of zero. In this space individual flanker values were drawn from Gaussian distribution with means $\left(m X^{j}\right)$ of $\pm 0.05,0.1,0.15$ and standard deviations $\left(s X^{j}\right)$ of $0,0.1,0.2$. Resampling of flanker feature values occurred until $m X^{j}$ and $s X^{j}$ fell within 0.01 from the desired values. Participants completed 10 blocks of 96 trials for this experiment.

Experiment 3 (fMRI study). All stimuli were presented on a grey background. Participants first saw a fixation dot for $500 \mathrm{~ms}$, followed by the presentation of stimuli for $500 \mathrm{~ms}$, and were asked to 
respond within 1500 ms of stimulus onset. Stimuli were tilted gratings as for Exp.1 and Exp.2a. At the end of response window, visual feedback in the form of a green or red dot indicated whether the response was correct or not. There was a jittered interval of between 2-6s (on average 4s) interposed between trials. Target tilt and flanker tilt statistics were varied independently on a trial-by-trial basis. On each trial, tilts of the target grating and flanker mean orientation were drawn independently from uniform distributions with a limit of $-45^{\circ}$ and $+45^{\circ}$. Similarly, flanker standard deviation could be values between 0 to $30^{\circ}$ from a uniform distribution. Resampling was used to ensure that the number of congruent and incongruent trials was matched across the entire experiment. Participants completed $4(n=10)$ or $5(n=10)$ blocks of the task in the scanner, with 130 trials on each block.

fMRI acquisition and preprocessing. Images were acquired with 3-Tesla Siemens TrioTim with a

32-channel head coil using a standard echo-planar imaging sequence. Images were $64 \times 64 \times 32$

505 volumes with voxel size $3.5 \times 3.5 \times 3.5 \mathrm{~mm}$; acquired with a $2 \mathrm{~s}$ repetition time and echo time of 30 ms. We acquired $\mathrm{fMRI}$ data in 4 or 5 runs with 425 volumes per run. All preprocessing and fMRI 507 analyses were carried out using SPM12. Preprocessing of the imaging data includes realignment of 508 function images, co-registration of anatomical scan to the mean functional image, followed by 509 segmentation and spatial normalisation to the standard template brain of the Montreal 510 Neurological Institute (MNI brain). Lastly function images were spatially smooth with a 6-mm full 511 width half maximum Gaussian kernel. A 128-s temporal high-pass filter was applied to exclude low-frequency artefacts.

\section{Design and behavioural analysis}


For experiments 1 and 2, the design orthogonalised the manipulation of target feature value $\left(X^{i}\right)$, mean of flankers $\left(m X^{j}\right)$ and variability of flankers $\left(s X^{j}\right)$. We can further designate trials as "congruent" when $X^{i}$ has the same sign as $m X^{j}$ or "incongruent" when $X^{i}$ has the opposite sign as

$518 m X^{j}$. In Experiment 1 , we thus have a $3 \times 2$ (flanker variability $\times$ congruency) within-participant 519 factorial design. In experiment 2, we introduced three levels of $\left|m X^{j}\right|$ and three levels of $\left|s X^{j}\right|$, 520 resulting in $3 \times 3 \times 3 \times 2\left(\left|X^{i}\right| \times\left|m X^{j}\right| \times s X^{j} \times\right.$ congruency) within-participant factorial design with 52154 conditions. Full list of $X^{i},\left|m X^{j}\right|$, and $s X^{j}$ levels were displayed in Table S1. For Exp. 1 and 2, 522 ANOVAS with Greenhouse-Geisser correction for sphericity were carried out at group-level 523 analyses. A threshold of $p<0.05$ was adopted for all behavioural analyses. We only analysed RT 524 from correct trials, and additionally excluded trials where RT was faster than the $1 \%$ percentile or slower than the $99 \%$ percentile of the RT distribution. We used the same exclusion criteria across experiments.

Computational modelling

Adaptive gain model. The computations that describe the population coding version of the

531 adaptive gain model are described in the main text. To fit model outputs to human response time 532 data (i.e. on a common scale in $\mathrm{ms}$ ), for each parameterisation we regressed inverse decision 533 values against each individual participants' response times:

$$
R T_{\text {gain }}=\beta^{0}+\beta^{1} \cdot 1 /\left|\hat{X}_{i}\right|
$$


538 This calculation of RT is equivalent to modelling the data with ballistic (noiseless) diffusion

539 process, with the two additional parameters $\beta^{0}$ and $\beta^{1}$ encoding the drift rate and non-decision

540 time respectively (fixed across conditions). Searching, exhaustively across values of $\sigma^{\max }$ and $\varepsilon$

541 from equation (2), we identified the parameters that minimised mean squared error (MSE)

542 between the human and model-predicted average RTs for each condition.

543

544 Conflict models. For the conflict model, we use a formulation described previously, whereby 545 conflict $C$ depends on the weighted product of competing inputs for the two actions $A^{C W}$ and

$546 A^{C C W}$

$$
\begin{gathered}
C=\left[A^{C W} \cdot A^{C C W}\right] \\
A^{C W}=g\left(m X^{j}\right) \cdot(1-w)+g\left(X^{i}\right) \cdot w \\
A^{C C W}=h\left(m X^{j}\right) \cdot(1-w)+h\left(X^{i}\right) \cdot w
\end{gathered}
$$

where $g(X)$ and $h(X)$ respectively denote positive and negative linear rectification functions. In conflict model 1 , activation for the two actions $A^{C W}$ and $A^{C C W}$ is proportional to the tilt of the each individual flanker (conflict model 2).

$$
C_{i}=\left[A_{i}^{C W} \cdot A^{C C W}\right]
$$

$$
A^{C W}=\sum_{1}^{j} g\left(X^{j}\right) \cdot \frac{(1-w)}{6}+g\left(X^{i}\right) \cdot w
$$




$$
A^{C C W}=\sum_{1}^{j} h\left(X^{j}\right) \cdot \frac{(1-w)}{6}+h\left(X^{i}\right) \cdot w
$$

564 Where $j$ is the number of flankers that are either congruent or incongruent. In other words, these models made different assumptions about conflict: that it was computed at the level of individual flankers (conflict model 2) or at the level of summary statistics (conflict model 1).

Finally, we compute RTs in a similar fashion as for the gain model:

$$
R T_{\text {conflict }}=\beta^{0}+\beta^{1} \cdot C
$$

573 To reduce the risk of overfitting we used cross-fitting, estimating model parameters on half the trials and computing mean squared error (MSE) for the other half. These MSE were then fed into Bayesian Model Selection (BMS) to compute exceedance probabilities.

Simulations of qualitative effects

578 When fit to human data, the adaptive gain model contained 2 free parameters: maximum tuning 579 width $\left(\sigma^{\max }\right)$ and sensory noise $(\varepsilon)$. Simulations of the model aimed at qualitatively recreating 580 effects from the past literature (e.g. for Fig.1, Fig. 6) assumed a fixed $\sigma^{\max }\left(\sigma^{\max }=10\right)$ and a 581 fixed $\varepsilon(\varepsilon=5)$ unless noted otherwise. 
Tilt illusion. In this simulation, we plot the difference between the true target angle (here, zero) and the gain modulated decision value $\left(\widehat{X^{\imath}}\right)$ as a function of flanker mean decision value $\left(m X^{j} \in\right.$ $\left.\left\{-45,-44, \ldots,+45^{\circ}\right\}\right)$ and flanker standard deviation $\left(s X^{j} \in\{3,7,11,15\}\right)$. For each variant of flanker mean decision values and flanker standard deviation, $\widehat{X^{\imath}}$ is computed using equation (1) and 2. We then plot $\widehat{X^{\imath}}$ against levels of flanker mean decision value and flanker standard deviation in Fig. 1a.

Conflict effects. We computed a proxy of RT $\left(1 / \widehat{X^{\imath}}\right)$ for the 3 conditions: 'CO': Congruent, where the target shares the same response association with the flankers; 'Sl': Stimulus Incongruent, where the target is perceptually different to the flankers but the response associations of the two are still the same; 'RI': Response Incongruent, where the target has a different response association as the flankers (Fig. 1b). In the simulation, flanker standard deviation $s X^{j}$ is set to be 0 in both ' $\mathrm{CO}$ ' and 'RI' condition (i.e. we assumed flanker standard deviation was $\varepsilon$, or $5^{\circ}$ ). In ' $\mathrm{CO}^{\prime}, X^{i}$ is equal to $m X^{j}$ (both are $\left.+45^{\circ}\right)$. In ' $\mathrm{Rl}^{\prime}, m X^{j}$ has the opposite sign to the $X^{i}$. Lastly, we simulated 'Sl' condition by assuming $s X^{j}$ is higher than 0 i.e. $s X^{j}=5$; individual flankers are variable but $m X^{j}$ remained the same as $X^{i}$. We assumed a higher maximum tuning width $\left(\sigma^{\max }=15\right)$ in this simulation.

Multi-alternative valued-based decision making task. In Fig. 1c, we simulated the difference 602 between the model estimated decision values from the two targets $\left(X^{i+}=20 \& X^{i-}=10\right)$ as a 603 function of a third distractors' orientation $\left(X^{j} \in\left\{-45,-44, \ldots,+45^{\circ}\right\}\right) . s X^{j}$ was assumed to be 5 604 in this simulation. In Fig. S1, we computed the model output associated with the highest-valued 605 choice-relevant alternative $\left(\widehat{X^{\imath+}}\right)$ and the next best alternative $\left(\widehat{X^{\imath-}}\right)$, assuming that the mode of the gain field determined by the statistics lowest-valued (i.e. irrelevant) alternative $X^{j}$. We then 
607 plotted a quantity proportional to choice probability, $\widehat{X^{\imath+}}-\widehat{X^{\imath-}}$, i.e. the relative difference in 608 model output for the best and next best options.

609

610 Value of chosen vs. unchosen option. We simulated the model output as a function of the value of

611 a theoretical chosen and unchosen option. On each trial, decision values for two stimuli $\left(X^{1}\right.$ and

$612 X^{2}$ ) were drawn independently from two zero-mean Gaussian distribution with a standard

613 deviation of 10. On every trial, we allowed simultaneous evaluation of each stimulus in the context

614 of the other, i.e. we passed each stimulus through the model as target with the alternative as

615 distracter. We then assumed that participants chose according to the relative subjective (i.e.

616 model output) value of the maximum and minimum resulting values, using a value of 5 for the

617 slope of the choice function:

$$
D=\hat{X}^{\max }-\hat{X}^{\min }
$$

$$
C P=\frac{1}{1+e^{\frac{-D}{5}}}
$$

622 This allowed us to plot the relationship between $X$ and model output $D$ separately for the chosen 623 and unchosen options.

625 Behavioural model comparison. We implemented Bayesian model selection at the group level to 626 obtain posterior likelihoods and exceedance probabilities as described by Stephan and 627 colleagues $^{63}$. 
631 Univariate Analyses. We analysed our data using Statistic Parametric Mapping (SPM12) with the 632 general linear model (GLM) framework and in-house scripts running in Matlab. For all analyses, we 633 ensured that sequential orthogonalisation of predictors in SPM was disabled. All GLMs also 634 included regressors encoding the estimated movement parameters from preprocessing as a 635 nuisance covariate. We modelled correct trials and trials that RT falls within 1 and 99 percentile 636 distribution. All other trials were modelled separately as a nuisance regressor in all GLMs. We first 637 constructed GLM1 with a single predictor encoding the parametric target decision values $\left|X^{i}\right|$ of 638 the stimulus, time-locked to the onset of the stimulus. We identified voxels activated negatively by 639 this regressor to define regions of interest in the $\mathrm{dACC}, \mathrm{AIC}$ and SPL. Activations in these regions 640 survived FDR correction for multiple comparisons at $p<0.05$. To avoid double-dipping for later 641 analyses, each region was identified in a leave-one-out fashion, with each participant in turn being 642 omitted from a group-level analysis, which was used to define a Region-of-Interest (ROI) with a 643 threshold of uncorrected $p<0.0001$, from which beta values were extracted from the left out 644 participant. For GLM2, we discretise each parametric modulator (i) target decision values $\left|X^{i}\right|$ and 645 (ii) flanker mean feature value $\mid m X^{j}$ |into 4 quartiles bins and included a total of $4 \times 4=16$ 646 regressors (corresponding to each quartile bins of feature values) in the GLM. BOLD betas from 647 each subject are extracted using each ROI masks defined by the leave-one-out analysis. In GLM3, 648 we entered the adaptive gain model estimates $1 /\left|\widehat{X}_{i}\right|$ and binary regressor (congruency: +1 for 649 congruent trials, -1 for incongruent trials) as competitive regressors. In GLM4, we replaced 650 congruency with response time as a competing regressor to $1 /\left|\hat{X}_{i}\right|$. In GLM5, we included the 651 following predictors as parametric modulators of the stimulus at the time of the decision (i) target 652 decision values $\left|X^{i}\right|$, (ii) flanker mean feature value $\left|m X^{j}\right|$, (iii) flanker precision, i.e. inverse 653 flanker variability $1 / s X^{j}$, (iv) absolute distance between target and flanker mean orientations 
$654\left|X^{i}-m X^{j}\right|,(\mathrm{v})$ the interaction of the distance between target and flanker mean with target

655 decision values $\left|X^{i}-m X^{j}\right| \times\left|X^{i}\right|$, and lastly (vi) the interaction of the distance between target 656 and flanker mean, target decision values and flanker variability $\left(\left|X^{i}-m X^{j}\right| \times\left|m X^{j}\right| \times 1 / s X^{j}\right)$. 657 Full details of active voxels associated with these regressor were displayed in Table S2-4. 658 Subsequent statistical tests on the betas extracted from each ROI were conducted using two-tailed 659 one-sample $t$-test at a significance threshold of $p<0.05$.

660

661 Neural Bayesian model comparison. We estimated the following GLMs using Bayesian statistics 662 and conducted model comparisons to quantify and compare the predictions of different GLMs: (i) 663 no-gain modulated decision value $\left|X^{i}\right|$ i.e. GLM1 described above, (ii) gain modulated decision 664 value $\left(\left|\hat{X}_{i}\right| ; \mathrm{GLM} 6\right), 3 \mathrm{GLMs}$ that contained the no-gain modulated decision value and conflict 665 defined in three ways (iii) GLM7: a binary congruency regressor (iv) GLM8: conflict computed using 666 equation (4) (v) GLM9: conflict computed using equation (5). The fits of each GLM were assessed 667 by Random Effects Bayesian Model Selection from the VBA toolbox ${ }^{50}$. This allows us to estimate 668 the exceedance probabilities and expected frequencies for each model by feeding the log 669 evidence for the aforementioned GLMs within each masks from the leave-one-out analysis. The 670 best model is characterised with the highest exceedance probability and an expected frequency 671 that is above chance (i.e. 1 /number of models in comparison $=20 \%$ ).

\section{References}

6741 Luce, R. D. Individual Choice Behavior: A Theoretical Analysis. (Wiley, 1959).

6752 von Neumann, J. \& Morgenstern, O. Theory of Games and Economic Behavior. (Princeton $676 \quad$ University Press, 1944).

6773 Savage, L. J. The Foundations of Statistics. (Wiley, 1954). 
6784 Wald, A. \& Wolfowitz, J. Bayes Solutions of Sequential Decision Problems. Proc Natl Acad 679 Sci U S A 35, 99-102 (1949).

6805 Ma, W. J., Beck, J. M., Latham, P. E. \& Pouget, A. Bayesian inference with probabilistic 681 population codes. Nat Neurosci 9, 1432-1438, doi:nn1790 [pii]

$68210.1038 / \mathrm{nn} 1790(2006)$.

6836 Kording, K. Decision theory: what "should" the nervous system do? Science 318, 606-610, 684 doi:10.1126/science.1142998 (2007).

6857 Blakemore, C., Carpenter, R. H. \& Georgeson, M. A. Lateral inhibition between orientation 686 detectors in the human visual system. Nature 228, 37-39 (1970).

6878 Eriksen, B. A. \& Eriksen, C. W. Effects of noise letters upon identification of a target letter in 688 a non- search task. Perception and Psychophysics 16, 143-149 (1974).

6899 Kopp, B., Mattler, U. \& Rist, F. Selective attention and response competition in 690 schizophrenic patients. Psychiatry Res 53, 129-139 (1994).

69110 Louie, K., Khaw, M. W. \& Glimcher, P. W. Normalization is a general neural mechanism for 692 context-dependent decision making. Proc Natl Acad Sci U S A, doi:1217854110 [pii]

693 10.1073/pnas.1217854110 (2013).

69411 Chau, B. K., Kolling, N., Hunt, L. T., Walton, M. E. \& Rushworth, M. F. A neural mechanism 695 underlying failure of optimal choice with multiple alternatives. Nat Neurosci 17, 463-470, doi:10.1038/nn.3649 (2014).

69712 Tsetsos, K., Usher, M. \& Chater, N. Preference reversal in multiattribute choice. 698 Psychological review 117, 1275-1293, doi:10.1037/a0020580 (2010).

69913 Tversky, A. Elimination by aspects: A theory of choice. Psychological review 79, 281-299 700 (1972). 
70114 Botvinick, M. M., Braver, T. S., Barch, D. M., Carter, C. S. \& Cohen, J. D. Conflict monitoring and cognitive control. Psychological review 108, 624-652 (2001).

70315 Bundesen, C. A computational theory of visual attention. Philos Trans R Soc Lond B Biol Sci 353, 1271-1281, doi:10.1098/rstb.1998.0282 (1998).

16 Heeger, D. J. Normalization of cell responses in cat striate cortex. Vis Neurosci 9, 181-197, doi:S0952523800009640 [pii] (1992).

17 Soltani, A., De Martino, B. \& Camerer, C. A range-normalization model of contextdependent choice: a new model and evidence. PLoS Comput Biol 8, e1002607, doi:10.1371/journal.pcbi.1002607

PCOMPBIOL-D-12-00223 [pii] (2012).

18 Schwartz, O., Sejnowski, T. J. \& Dayan, P. Perceptual organization in the tilt illusion. J Vis 9, 19 11-20, doi:10.1167/9.4.19 (2009). 2248-2260, doi:10.1016/j.visres.2010.04.014 (2010). based attention. Psychological review 103, 603-649 (1996).

21 Yu, A. J., Dayan, P. \& Cohen, J. D. Dynamics of attentional selection under conflict: toward a rational Bayesian account. J Exp Psychol Hum Percept Perform 35, 700-717, doi:10.1037/a0013553 (2009). cortex. J Neurosci 29, 14004-14014, doi:29/44/14004 [pii] 1441, doi:10.1016/j.neuron.2014.01.020 (2014). 
72524 Simoncelli, E. P. \& Olshausen, B. A. Natural image statistics and neural representation. Annual review of neuroscience 24, 1193-1216, doi:10.1146/annurev.neuro.24.1.1193 (2001).

25 Rangel, A. \& Clithero, J. A. Value normalization in decision making: theory and evidence. Current opinion in neurobiology 22, 970-981, doi:10.1016/j.conb.2012.07.011 (2012).

Kolling, N. et al. Value, search, persistence and model updating in anterior cingulate cortex. Nat Neurosci 19, 1280-1285, doi:10.1038/nn.4382 (2016).

Shenhav, A., Cohen, J. D. \& Botvinick, M. M. Dorsal anterior cingulate cortex and the value of control. Nat Neurosci 19, 1286-1291, doi:10.1038/nn.4384 (2016).

Heilbronner, S. R. \& Hayden, B. Y. Dorsal Anterior Cingulate Cortex: A Bottom-Up View. Annual review of neuroscience 39, 149-170, doi:10.1146/annurev-neuro-070815-013952 (2016).

Michael, E., de Gardelle, V. \& Summerfield, C. Priming by the variability of visual information. Proc Natl Acad Sci U S A 111, 7873-7878, doi:10.1073/pnas.1308674111 (2014).

Solomon, J. A. \& Morgan, M. J. Stochastic re-calibration: contextual effects on perceived tilt. Proc Biol Sci 273, 2681-2686, doi:10.1098/rspb.2006.3634 (2006).

31 Gibson, J. J. Adaptation with negative aftereffect. Psychological review 44, 222-244 (1937).

Wu, T., Dufford, A. J., Mackie, M. A., Egan, L. J. \& Fan, J. The Capacity of Cognitive Control Estimated from a Perceptual Decision Making Task. Scientific Reports 6 (2016). 

averaging protects decisions from noise in neural computations. PLoS Comput Biol 13(8): e1005723. https://doi.org/10.1371/journal.pcbi.1005723

Van Veen, V. \& Carter, C. S. The timing of action-monitoring processes in the anterior cingulate cortex. Journal of cognitive neuroscience 14, 593-602 (2002).

36 Carter, C. S. et al. Science. Science 280, 747-749 (1998).

37 Kerns, J. G. et al. Anterior cingulate conflict monitoring and adjustments in control. Science 303, 1023-1026 (2004).

38 Ho, T. C., Brown, S. \& Serences, J. T. Domain general mechanisms of perceptual decision making in human cortex. J Neurosci

29, 8675-8687, doi:29/27/8675 [pii] 10.1523/JNEUROSCI.5984-08.2009 (2009).

39 Grinband, J., Hirsch, J. \& Ferrera, V. P. A neural representation of categorization uncertainty in the human brain. Neuron 49, 757-763, doi:S0896-6273(06)00089-4 [pii] 10.1016/j.neuron.2006.01.032 (2006).

Wunderlich, K., Rangel, A. \& O'Doherty, J. P. Neural computations underlying action-based decision making in the human brain. Proceedings of the National Academy of Sciences 106, 17199-17204 (2009).

41 Tsetsos, K., Wyart, V., Shorkey, S. P. \& Summerfield, C. Neural mechanisms of economic commitment in the human medial prefrontal cortex. eLife 3, e03701 (2014).

42 Juechems, K., Balaguer, J., Ruz, M. \& Summerfield, C. Ventromedial Prefrontal Cortex Encodes a Latent Estimate of Cumulative Reward. Neuron 93, 705-714 (2017).

43 Kolling, N., Behrens, T. E., Mars, R. B. \& Rushworth, M. F. Neural mechanisms of foraging. 
77044 Barber, A. D. \& Carter, C. S. Cognitive control involved in overcoming prepotent response tendencies and switching between tasks. Cerebral Cortex 15, 899-912 (2004).

Rushworth, M. F., Noonan, M. P., Boorman, E. D., Walton, M. E. \& Behrens, T. E. Frontal cortex and reward-guided learning and decision-making. Neuron 70, 1054-1069, doi:S08966273(11)00395-3 [pii] 10.1016/j.neuron.2011.05.014 (2011). theory of anterior cingulate cortex function. Neuron 79, 217-240 (2013). anterior cingulate cortex. Current opinion in neurobiology 37, 36-43 (2016).

Shahnazian, D. \& Holroyd, C. B. Distributed representations of action sequences in anterior cingulate cortex: A recurrent neural network approach. Psychonomic Bulletin \& Review, 120 (2017).

Penny, W., Mattout, J. \& Trujillo-Barreto, N. in Statistical Parametric Mapping: The analysis of functional brain images (eds K. Friston et al.) (Elsevier, 2006).

50 Daunizeau, J., Adam, V. \& Rigoux, L. VBA: a probabilistic treatment of nonlinear models for neurobiological and behavioural data. PLoS computational biology 10, e1003441. (2014).

51 Grinband, J. et al. The dorsal medial frontal cortex is sensitive to time on task, not response conflict or error likelihood. Neuroimage 57, 303-311, doi:S1053-8119(10)01610-1 [pii] 10.1016/j.neuroimage.2010.12.027 (2011). versus selection-for-action in anterior cingulate cortex. Nature 402, 179-181, doi:10.1038/46035 (1999). 
Alternative Choice. J Neurosci

33, 2242-2253,

doi:33/6/2242

[pii] 10.1523/JNEUROSCI.3022-12.2013 (2013).

Hazeltine, E., Poldrack, R. \& Gabrieli, J. D. Neural activation during response competition. J Cogn Neurosci 12 Suppl 2, 118-129, doi:10.1162/089892900563984 (2000).

Gratton, G., Coles, M. G. \& Donchin, E. Optimizing the use of information: strategic control of activation of responses. J Exp Psychol Gen 121, 480-506 (1992).

80056 Boorman, E. D., Behrens, T. E., Woolrich, M. W. \& Rushworth, M. F. How green is the grass on the other side? Frontopolar cortex and the evidence in favor of alternative courses of action.

Neuron

62, 733-743

doi:S0896-6273(09)00389-4

[pii] 10.1016/j.neuron.2009.05.014 (2009).

Egner, T. \& Hirsch, J. Cognitive control mechanisms resolve conflict through cortical amplification of task-relevant information. Nat Neurosci 8, 1784-1790, doi:nn1594 [pii] 10.1038/nn1594 (2005).

Stemmler, M., Usher, M. \& Niebur, E. Lateral interactions in primary visual cortex: a model bridging physiology and psychophysics. Science 269, 1877-1880 (1995). Eimer, M. Facilitatory and inhibitory effects of masked prime stimuli on motor activation and behavioural performance. Acta Psychol (Amst) 101, 293-313 (1999). Percept Psychophys 65, 287-297 (2003). 10.1038/nn1954 (2007). 
81763 Stephan, K. E., Penny, W. D., Daunizeau, J., Moran, R. J. \& Friston, K. J. Bayesian model selection for group studies.

Neuroimage

46, 1004-1017, doi:10.1016/j.neuroimage.2009.03.025 (2009).

\section{Author Contributions}

822 V.L., E.M. and C.S. conceived the study and designed the experiments; V.L., J.B. and S.H.C.

823 collected data., V.L. and C.S. analysed the data, J.B. contributed custom scripts for fMRI data

824 analysis. V.L. and C.S. wrote the manuscript, with helpful input from the other authors.

\section{Acknowledgements}

827 We thank Tobias Egner for comments on the early version of the manuscript. This work was

828 funded by European Research Council (ERC) Starter Grant (281628) to C.S., Croucher Foundation

829 scholarship to V.L., Medical Research Council studentship to E.M. and the Wellcome Trust

$830(0099741 /$ Z/12/Z) to S.H.C. All funders had no role in the conceptualization, design, data

831 collection, analysis, decision to publish, or preparation of the manuscript.

\section{Competing Interests}

834 The authors declare no competing interests.

\section{Data Availability}

837 All behavioural data are made available as Supplementary Information. Request for custom code and $\mathrm{fMRI}$ data should be directed to the corresponding author 